EPJ manuscript No.

(will be inserted by the editor)

\title{
Introduction to colloidal dispersions in external fields
}

\section{H. Löwen}

Institut für Theoretische Physik II: Weiche Materie, Heinrich-Heine-Universität Düsseldorf, 40225 Düsseldorf, Germany

\begin{abstract}
Progress in the research area of colloidal dispersions in external fields within the last years is reviewed. Colloidal dispersions play a pivotal role as model systems for phase transitions in classical statistical mechanics. In recent years the leading role of colloids to realize model systems has become evident not only for equilibrium situations but also far away from equilibrium. By using external fields (such as shear flow, electric, magnetic or laseroptical fields as well as confinement), a colloidal suspension can be brought into nonequilibrium in a controlled way. Various kinds of equilibrium and nonequilibrium phenomena explored by colloidal dispersions are described providing also a guide and summary to this special issue. Particular emphasis is put on the comparison of real-space experiments, computer simulations and statistical theories.
\end{abstract}

\section{Introduction}

The special topic of this themed issue is the physics of colloidal dispersions which is a rapidly growing research area in the interdisciplinary soft matter domain. Colloidal dispersions are solutions of mesoscopic solid particles with typical sizes ranging from 1-10 $\mathrm{nm}$ to about $1-10 \mu \mathrm{m}$ with a stable (i.e. non-fluctuating) core embedded in a molecular fluid solvent. Among the various soft matter systems, colloidal dispersions play a pivotal role as model systems, as they can be both prepared and characterized in a controlled way. The effective interaction between the colloidal particles can be tailored by changing, e.g., the salt concentration in the solvent. Moreover, colloidal suspensions can be regarded as the simplest prototype of soft matter: the length scale separation between the molecular solvent and the mesoscopic particles is unique and complete. Spherical particles without any additional structure on the mesoscopic length scale possess the simplest and highest possible symmetry. This directly implies that a simple theoretical modelling of a single particle without many fitting parameters is possible. Exciting questions concern collective many-body effects induced by cooperation and self-organization of many particles, in particular for bulk phase transitions such as fluid-fluid phase separation, freezing as well as glass and gel formation.

As an example, Fig. 11 shows an electron micrograph of mesoscopic colloidal particles which look really like spheres on the micron scale. What can be directly seen from Fig. 1 is that the particles form crystalline lattices with a mesoscopic lattice constant. Correspondingly, the time scale upon which crystallization occurs is slow enough such 


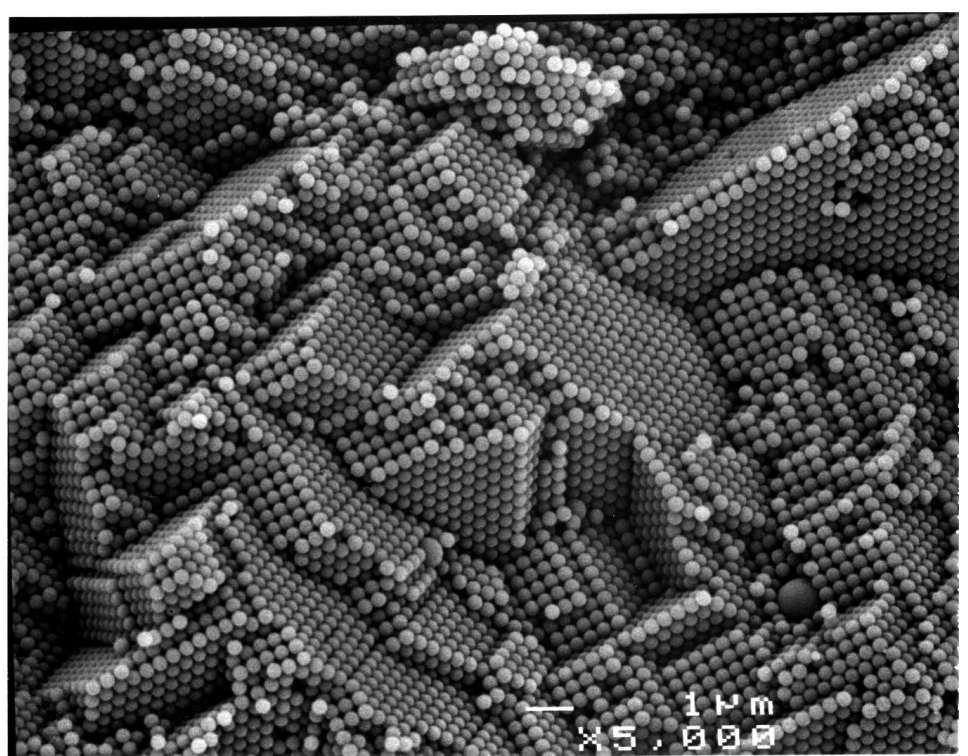

Fig. 1. Real-space image of submicron colloidal PMMA spheres will exhibit long-range positional order on a crystalline lattice ${ }^{2}$

that configurational changes can be studied by tracking the individal particle trajectories. Therefore the full "microscopic", i.e. particle-resolved, information is available, similar as in classical particle-resolved computer simulations.

Moreover, the properties of colloidal dispersions can efficiently be tailored and controlled by employing external fields. The study of of an external control via external fields has two main reasons:

1. First, by definition, soft matter reacts sensitively ("softly") upon external perturbations and manipulations. The occurrence of stable colloidal bulk samples is the exception rather than the rule, i.e., one has to protect the sample carefully against shear and other perturbations. How protection can work needs therefore detailed study.

2. The second reason is that strong external fields induce qualitatively novel effects not known from equilibrium bulk. Complementarily, the softness is rather exploited than being protected in this case.

An external field can either give rise to an equilibrium problem (e.g. by modifying the interparticle interactions) or drives the system into nonequilibrium. Colloidal dispersions in external fields play therefore a similar pivotal role as model systems for controlled nonequilibrium situations. This opens the way for a fundamental understanding of nonequilibrium phenomena which is important also for technological applications. Significant achievements in understanding the principles of the behaviour in non-equilibrium were gained by a systematic comparison of results from experiment, theory and computer simulation. In this special issue, the structural and dynamical behaviour of colloidal dispersions in various external fields are discussed both in equilibrium and in non-equilibrium. The external fields can be:

- shear flow

- electric fields

\footnotetext{
${ }^{2}$ Taken from http://www.physics.nyu.edu/pine/Pine___Res_-_Clusters.html
} 
- laser-optical and magnetic fields

- confining geometries

During the last years, significant developments have stimulated the colloid research in general and colloidal physics in external fields in particular. First of all, qualitative novel behaviour genuinely induced by an applied field has been discovered in theory and experiment leading to new nonequilibrium phenomena. Second, different experimental techniques have been applied to study colloidal dispersions, in particular real space techniques. Next, computer simulation schemes have been applied and developed, e.g. in order to simulate hydrodynamic interactions between the colloidal particles mediated by the solvent. Last but not least, new statistical theories for colloidal models have been developed and significantly extended.

The major motivation is a microscopic understanding - on the basic time- and length scales - of the particles in non-equilibrium. The route toward this challenging goal can be best explained in terms of complexity. The level of different complexities is classified and summarized in the traditional complexity diagram of Fig. 2 [1. On the horizontal axis, the degree of complexity of the system is shown related to the statistical degrees of freedom present in the system. The simplest case are spherical particles while mixtures and orientational degrees of freedom represent a higher level of system complexity. Even higher complexity is arising from particles with a nonconvex shape like colloidal molecules or with internal degrees of freedom, e.g. with an internal motor [2]3. On the other hand, the complexity of the question asked which is shown on the vertical axis can comprise equilibrium situations, such as inhomogeneous systems near walls and in restricted geometries, steady-state non-equilibrium cases (such as systems under permanent shear flow or other time- independent or oscillatory external fields). Finally, full non-equilibrium situations arise if a field is turned on or switched off. A thorough understanding can only be achieved via a systematic, step-by-step process in the complexity diagram as indicated by the arrows in Figure 2. While the covering of the unknown part was a vision more than 10 years ago when the complexity diagram was first proposed [1], this vision has now become true and was realized as documented by the research results contained in this special issue.

In this introductory article, recent highlights obtained in our understanding of colloidal dispersions in various external fields will be briefly presented. First we shall consider four different types of external fields consecutively, namely shear flow, electric, laser-optical and magnetic fields as well as confinement. Then we shall give an outlook about future research activities in the realm of colloid physics where we shall address anisotropic colloids and self-propelled particles with intrinsic degrees of freedom, in particular. This article therefore provides also a brief classification of the 23 following minireviews contained in this special issue.

A large portion of the results discussed here were obtained within the GermanDutch Collaborative Research Centre SFB TR6 with the title Physics of Colloidal Dispersions in External Fields within the last funding period 2009-2013. The SFB TR6 network comprises the locations Düsseldorf, Jülich, Konstanz, Mainz and Utrecht but has various collaborations with more external project partners.

As a general final remark, colloidal dispersions show strong analogies to complex plasmas which are similar form-stable mesoscopic particles embedded in a plasma. The similarities and complementarities were recently discussed, see e.g. 4 for an overview. Examples include separation kinetics in binary mixtures [5] and lane formation [6] which are found both in colloids and in complex plasmas but with different details in the dynamics. Another fruitful link is to granular materials [8] which allow for a direct observation of the particles but do not exhibit Brownian motion. 


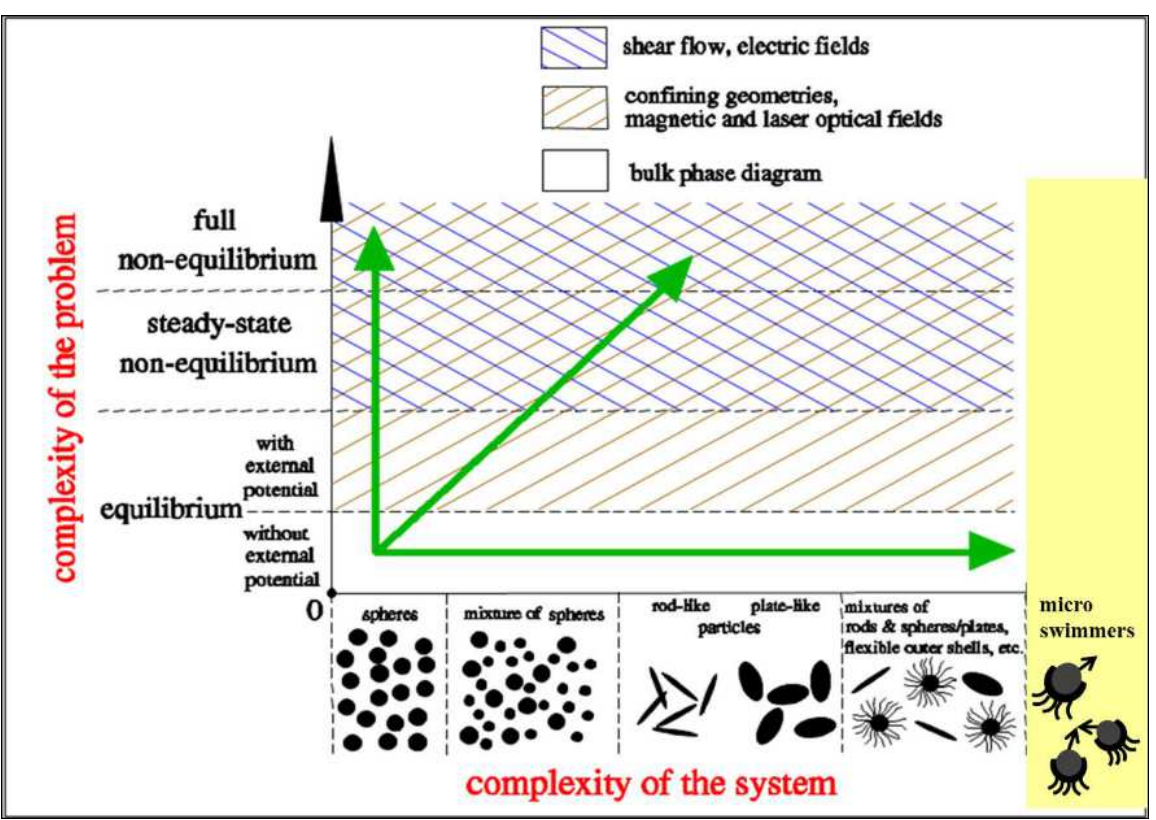

Fig. 2. Road map of complexity for colloidal dispersions in external fields: while the x-axis shows the complexity of the system, the y-axis shows the complexity of the problem. Regions which can be accessed by different kinds of external fields are indicated. The arrows indicate recent research directions. Active particles or microswimmers, which exhibit a special complexity induced by their internal propulsion, are also indicated.

\section{Shear fields}

A shear field naturally occurs for colloidal dispersions in a solvent flow. In the past years, there has been a significant progress in understanding sheared colloidal suspensions within a microscopic or schematic mode-coupling-like theory, in particular close to the glass transition. For example, a schematic model for the constitutive rheology of glasses was presented [9]. The resulting tensorial structure of the schematic model satisfies applicable invariance laws for the nonlinear flow of materials without inertia, such as colloidal suspensions. As a concrete example, the full dynamic yield stress manifold was calculated by addressing a family of steady flows that interpolate between planar and uniaxial elongation. The resulting yield surface is quite similar, but not identical, to the empirical form of von Mises that has been widely used to model static yield and plasticity in solids. This theory thus gives a first-principle justification for this empirical law. A variant of this theory was tested against nonlinear rheology data of concentrated thermosensitive core-shell particles and excellent agreement was found concerning yielding, shear-thinning and large amplitude oscillatory shearing [10]. More aspects are reviewed in [11.

Soft particles deform under shear and their structural and dynamical behaviour was intensely studied both by experiment [12] and theory [13. For instance, the dynamical deformation of ultrasoft colloids as well as their dynamic frictional forces were numerically investigated, when one colloid is dragged past another at constant velocity [14. Hydrodynamic interactions are captured by a particle-based mesoscopic simulation method. At large drag velocities, an apparent attractive force was found for departing colloids along the dragging direction.

Transient dynamics after switching on shear was obtained by a joint venture of theory, simulation and experiment, see e.g. 15. In particular, concentrated hard- 
sphere suspensions and glasses were investigated with rheometry, confocal microscopy, and Brownian dynamics simulations during start-up shear, providing a link between microstructure, dynamics, and rheology [16]. The microstructural anisotropy is manifested in the extension axis where the maximum of the pair-distribution function exhibits a minimum at the stress overshoot. The interplay between Brownian relaxation and shear advection as well as the available free volume determine the structural anisotropy and the magnitude of the stress overshoot.

Colloids provide the fascinating possibility to drag single particles through the suspension, which gives access to microrheology (as opposed to macrorheology where macroscopic boundaries are moved). Several theoretical aspects of microrheology were discussed [171819] including again a schematic model of mode-coupling theory [20]. The latter model describes the strongly nonlinear behavior of the microscopic friction coefficient as a function of applied external force in terms of a delocalization transition, which is visible in Brownian dynamics simulations of a system of quasi-hard spheres and experimental data on hard-sphere-like colloidal suspensions. Moreover, molecular dynamics computer simulation of a glass-forming Yukawa mixture was used to study the anisotropic dynamics of a single particle pulled by a constant force [21]. Beyond linear response, a scaling regime was found where a force-temperature superposition principle holds. This is summarized in the minireviews $[22[23]$.

Finally, total Internal Reflection Fluorescence Cross-Correlation Spectroscopy uses fluorescent colloidal particles to probe the properties of a flow field near a surface 24. Careful experiments combined with a detailed theoretical analysis employing large-scale computer simulations are able to measure properties like the slip length accurately. However, the results also clearly show that the method requires detailed modeling of the diffraction phenomena at the confocal microscope's objective, and that this aspect needs further improvement.

Finally anisotropic particles show an even more complex response to shear flow as isotropic ones. Some recent findings are summarized in [25].

\section{Electric fields}

If a charged colloidal particle is placed into an electric field it gets into motion. This electrokinetic effect is, however, nontrivial due to the presence of counterions in the solution and due to solvent flow effects. In fact, electrophoresis constitutes already a nontrivial problem for a single colloidal particles since electrostatics and hydrodynamics couple in a complex way. Accordingly, a collective ensemble of charged colloids responds in an even more complicated way to an external electric field. In this special issue, electrokinetics will be accessed by experimental studies [26 27. by computer simulations [2829] and by statistical theory [30.

An electric field can also be used to manipulate and steer the self-assembly of colloids. This idea is in particular useful for nonspherical particles. Applications of these methods are discussed in 31 .

Finally we mention that binary mixtures of oppositely charged colloids can also be prepared corresponding to a mesoscopic realization of a molecular molten salt 32. The advantage of the colloidal system is that arbitrary charge and size ratios can be realized. This mixture exhibits fascinating bulk phase diagrams with a wealth of different mixed crystals [4. When placed into a DC electric field the oppositely charged particles will move against each other. At high enough field strength, lane formation of like-charge particles occurs 333234.

More recently, real-space experiments, computer simulation and analytic theory were performed for lane formation of oppositely charged colloids in electric fields 735 36]. The results demonstrate a continuous increase in the fraction of particles 
in a lane in the case that oppositely charged particles are driven by an electric field. This behavior is accurately captured by Brownian dynamics simulations. By studying the fluctuations parallel and perpendicular to the field a mechanism was identified that underlies the formation of lanes. In an AC-electric field, bands perpendicular to the field directions form. The detailed nonequilibrium phase diagram depends on the amplitude and frequency of the applied field as predicted by computer simulations [37. The banding effect was confirmed by real-space experiments [38. However, a detailed understanding of the hydrodynamic interactions between colloids 39134 is still lying ahead.

\section{Laser-optical and magnetic fields}

Laser-optical fields can be used to tailor a random substrate potential for colloids 40] or to bind colloids optically [41. A modulated substrate potential can be created by superimposing various laser fields and then the phase transitions of colloids in these periodic substrates can be explored where, in general, a large deviation from the bulk behaviour is found. The minireview [42] of this special issue summarizes some important examples.

External magnetic fields are typically used to create dipolar repulsions of colloids pending at an air-water interface. This provides an avenue to two-dimensional systems, where the freezing transition [43] and various transport phenomena through channels are in the focus of recent research [445]. One example is the realization of a sudden quench which can be applied to binary mixtures of superparamagnetic colloidal particles confined at a two-dimensional water-air interface by a sudden increase of an external magnetic field [46. This quench realizes a virtually instantaneous cooling which is impossible in molecular systems. Another example are two dimensional fluids with quenched disorder as realized by particles pinned to the substrate which show significant deviation in phase behavior as compared to pure systems 47 .

Various aspects of the impact of magnetic fields on colloids is intensely discussed in five minireviews of this special issue: The motion of magnetic particles in external potential barriers [48, and in time-dependent magnetic fields [49], the superposition of a magnetic field with shear flow [50] and gravity [51] and the extension from onecomponent systems to binary mixtures [51].

\section{Confinement}

The classic set-up of confinement is a slit-geometry realized by squeezing the suspensions between two parallel glass plates. The plate separation can easily be an interparticle spacing such that the effects of confinement are drastic as compared to the bulk. An important question concerns the nature of bulk phase transition like freezing, condensation or the glass transition in confinement.

Colloidal crystal confined in slit geometry have structures widely different from their bulk. A survey of recent developments can be found in the minireview [52. Examples include multilayered structures 32 and new structures solely induced by confinement [5354]. Also the dynamics of crystallization in confinement has been studied in detail, for example under gravity [54] and in a flow-field [55.

The dynamics of sedimentation of an initially inhomogeneous distribution of hardsphere colloids confined in a slit was studied by simulation and real-space microscopy experiments [56 57]. The scenario of the observed Rayleigh-Taylor-like instability depends crucially on the hydrodynamic interactions mediated by the solvent. 
The glass transition in slit geometry was calculated by extending mode-coupling theory, a microscopic theory for the glass transition of liquids [58. The glass transition line was calculated as a function of the distance of the plates for the case of a hard sphere fluid and an oscillatory behavior was obtained as a result of the structural changes related to layering.

In cylindrical confinement, computer simulations of colloid-polymer mixtures [59] have shown the existence of two distinct rounded transitions for condensation. The transition from multi-domain to a single domain state explains the hitherto mysterious "hysteresis critical point". Moreover, helical crystalline structures were predicted in cylindrical confinement 60.

Colloids can also be confined to liquid-gas interface. Capillary waves then lead to a mutual lateral attraction between the colloidal particles. The actual form of the attractive force is formally analogous to two-dimensional screened Newtonian gravity with the capillary length as the screening length. Therefore the set-up has the same equations as cosmology albeit on a much smaller length scale. The "gravitational" collapse was recently explored using Brownian dynamics simulations, density functional theory, and analytical perturbation theory 61, see also the minireview 62 .

Finally, anisotropic particles in confinement possess even more possibilities of structural phase transitions due to a competition of many length scales. Some aspects are reviewed in the subsequent papers $63,64,65$.

\section{Non-spherical particle shapes}

By now a plethora of non-spherical particle shapes can be prepared, see 4 for a recent review. Frequently these particles are called "colloidal molecules", in analogy to the traditional molecules in the microscopic regime. The advantage of the colloids relative to real molecules, however, is that arbitrary shapes can be principle be prepared at wish.

Here we mention three key examples: first, the phase behaviour of boardlike colloids as realized by goethite (a-FeOOH) particles were explored and biaxial nematic and biaxial smectic phases were found 66. The macroscopic domains were oriented by a magnetic field and their structure was revealed by small angle x-ray scattering. Second, particles with a different chirality (enantiomers) were considered 67. For many applications it is necessary to separate particles with different chirality. Such a separation can be achieved in microfluidic or nanofluidic channels if a solvent flow field is used which breaks chiral symmetry and has regions with high local shear 67. Such flow profiles can be generated in channels confined by walls with different hydrodynamic boundary conditions (e.g., slip lengths). Due to a nonlinear hydrodynamic effect, particles with different chirality migrate at different speed and can be separated.

Third, the theory of Brownian dynamics though well-developed for spheres and rods was completely formulated for interacting rigid biaxial particles with an arbitrary shape [6869]. Regarding more recent advances for anisotropic particles we refer the reader to the papers 6365 of this special issue.

\section{Active Colloids}

In contrast to passive particle in an external field, self-propelled particles dissipate energy and move autonomously. Their propagation direction is thereby an inner degree of freedom which is not only subjected to thermal fluctuations, the external field and 
particle interaction, as opposed to passive particles. This leads to intrinsic nonequilibrium behaviour 23. Self-propelled colloidal particles have been prepared where the external field again plays a leading role for the self-propagation. As prominent examples we mention catalytically-driven Janus-particles [70] and thermally driven colloids in a phase-separating solvent 7172,73 .

Self-propelled rod-like particles exhibit clustering which is not present in equilibrium 7475. They can be efficiently caught in a wedge-like trap [76 and show turbulence-like behaviour at intermediate densities [77. This turbulent behaviour occurs at low-Reynolds number in contrast to the traditional turbulence which happens at high Reynolds number.

Also the freezing transition has been studied for self-propelled colloids 78. The transition differs significantly from bulk freezing. The transition is accompanied by pronounced structural heterogeneities. This leads to a transition region between liquid and solid in which the suspension is globally ordered but unordered liquidlike "bubbles" still persist. For large propagation speed there is a transition from a resting crystals to a travelling crystal which migrates collectively [79. In terms of theory, the microscopic density functional approach has been formulated also for active systems 69. Finally, the articles 6348] of this special issue discuss more recent findings for self-propelled colloidal particles.

\section{Conclusions}

Meanwhile colloidal suspensions are not only excellent model systems to understand bulk phase transitions on the particle scale. The past decade has shown that colloids also provide principle guidance to understand and classify nonequilibrium behaviour as induced by external fields. The present special issue discusses a plethora of examples for field-induced effects in colloidal dispersions. New research typically goes hand-inhand by using complementary methods, namely real-space experiments, computer simulations and statistical theories.

The possibility of synthesize novel particle shapes and to prepare self-propelled colloidal particles has given two recent major boost for the research field of colloids. The collective behaviour of-non-spherical, self-propelled and active particles [80] is expected to become a flourishing field for the future.

Discussion with all members of the SFB TR6 are acknowledged. This work was supported by the DFG within SFB TR6.

\section{References}

1. H. Löwen, J. Phys.: Condens. Matter 13, R415 (2001).

2. P. Romanczuk, M. BÄr, W. Ebeling, B. Lindner, and L. Schimansky-Geier, Eur. Phys. J. Special Topics 202, 1 (2012).

3. M. E. Cates, Reports on Progress in Physics 75, 042601 (2012).

4. A. Ivlev, H. Löwen, G. Morfill, and C. P. Royall, Complex Plasmas and Colloidal Dispersions: Particle-Resolved Studies of Classical Liquids and Solids, Series in Soft Condensed Matter, World Scientific, 2012.

5. A. Wysocki, C. Raeth, A. V. Ivlev, R. K. Sütterlin, H. M. Thomas, S. A. Khrapak, S. K. Zhdanov, V. E. Fortov, A. M. Lipaev, V. I. Molotkov, O. F. Petrov, H. Löwen, and G. E. Morfill, Phys. Rev. Lett. 105, 045001 (2010).

6. R. K. R. K. Sütterlin, A. Wysocki, A. V. Ivlev, C. Raeth, H. M. Thomas, M. Rubin-Zuzic, W. J. Goedheer, V. E. Fortov, A. M. Lipaev, V. I. Molotkov, O. F. Petrov, G. E. Morfill, and H. Löwen, Phys. Rev. Lett. 102, 085003 (2009). 
7. T. Vissers, A. Wysocki, M. Rex, H. Löwen, C. P. Royall, A. Imhof, and A. van BlaAderen, Soft Matter 7, 2352 (2011).

8. I. S. Aranson and L. S. Tsimring, Rev. Mod. Phys. 78, 641 (2006).

9. J. M. Brader, T. Voigtmann, M. Fuchs, R. G. Larson, and M. E. Cates, Proc. Natl. Acad. Sci. USA 106, 15186 (2009).

10. M. Siebenbürger, M. Fuchs, and M. Ballauff, Soft Matter 8, 4014 (2012).

11. K. J. Mutch, M. Laurati, C. P. Amann, M. Fuchs, and S. U. EgelhaAf, Eur. Phys. J. Special Topics (accepted) .

12. S. K. Kundu, S. Gupta, J. Stellbrink, L. Willner, and D. Richter, Eur. Phys. J. Special Topics (accepted) .

13. R. G. Winkler, S. P. Singh, C.-C. Huang, D. A. Fedosov, K. Mussawisade, A. Chatterji, M. Ripoll, and G. Gompper, Eur. Phys. J. Special Topics (accepted)

14. S. P. Singh, R. G. Winkler, and G. Gompper, Phys. Rev. Lett. 107, 158301 (2011).

15. M. Laurati, K. J. Mutch, N. Koumakis, J. Zausch, C. P. Amann, A. B. Schofield, G. Petekidis, J. F. Brady, J. Horbach, M. Fuchs, and S. U. EgelhaAf, J. Phys.: Condens. Matter 24, 464104 (2012).

16. N. Koumakis, M. Laurati, S. U. Egelhaaf, J. F. Brady, and G. Petekidis, Phys. Rev. Lett. 108, 098303 (2012).

17. C. J. Harrer, D. Winter, J. Horbach, M. Fuchs, and T. Voigtmann, J. Phys.: Condens. Matter 24, 464105 (2012).

18. R. J. DePuit and T. M. Squires, J. Phys.: Condens. Matter 24, 464106 (2012).

19. R. J. DePuit and T. M. Squires, J. Phys.: Condens. Matter 24, 464107 (2012).

20. M. V. Gnann, I. Gazuz, A. M. Puertas, M. Fuchs, and T. Voigtmann, Soft Matter 7, 1390 (2011).

21. D. Winter, J. Horbach, P. Virnau, and K. Binder, Phys. Rev. Lett. 108, 028303 (2012).

22. A. Winkler, D. Winter, P. Chaudhuri, A. Statt, P. Virnau, J. Horbach, and K. Binder, Eur. Phys. J. Special Topics (accepted) .

23. T. Voigtmann and M. Fuchs, Eur. Phys. J. Special Topics (accepted) .

24. R. Schmitz, S. Yordanov, H. J. Butt, K. Koynov, and B. Dünweg, Phys. Rev. E 84, 066306 (2011).

25. D. Guu, J. K. G. Dhont, and M. P. Lettinga, Eur. Phys. J. Special Topics (accepted)

26. T. Palberg, H. Schweinfurth, T. Köller, H. Müller, H. J. SchöPe, and A. Reinmüller, Eur. Phys. J. Special Topics (accepted) .

27. J. Zhao, P. Papadopoulos, M. Roth, C. Dobbrow, E. Roeben, A. Schmidt, H.-J. Butt, G. K. Auernhammer, and D. Vollmer, Eur. Phys. J. Special Topics (accepted)

28. R. Schmitz, V. Starchenko, and B. Dünweg, Eur. Phys. J. Special Topics (accepted)

29. J. Zhou and F. Schmid, Eur. Phys. J. Special Topics (accepted).

30. G. Nägele, M. Heinen, A. J. Banchio, and C. Contreras-Aburto, Eur. Phys. J. Special Topics (accepted).

31. A. van BlaAderen, M. Dijkstra, R. van Roij, A. Imhof, M. Kamp, B. KwaAdgras, T. Vissers, and B. Liu, Eur. Phys. J. Special Topics (accepted) .

32. M. E. Leunissen, C. G. Christova, A.-P. Hynninen, C. P. Royall, A. I. Campbell, A. Imhof, M. Dijkstra, R. van Roij, and A. van BlaAderen, Nature 437, 235 (2005).

33. J. Dzubiella, G. P. Hoffmann, and H. Löwen, Phys. Rev. E 65, 021402 (2002).

34. H. LöWEn, Soft Matter 6, 3133 (2010).

35. M. Kohl, A. V. Ivlev, P. Brandt, G. E. Morfill, and H. Löwen, J. Phys.: Condens. Matter 24, 464115 (2012).

36. T. Glanz and H. Löwen, J. Phys.: Condens. Matter 24, 464114 (2012).

37. A. Wysocki and H. Löwen, Phys. Rev. E 79, 041408 (2009).

38. T. Vissers, A. van Blaaderen, and A. Imhof, Phys. Rev. Lett. 106, 228303 (2011). 
39. A. Wysocki and H. Löwen, J. Phys.: Condens. Matter 23, 284117 (2011).

40. M. Mazilu, A. Rudhall, E. M. Wright, and K. Dholakia, J. Phys.: Condens. Matter 24, 464117 (2012).

41. P. Dillmann, G. Maret, and P. Keim, J. Phys.: Condens. Matter 24, 464118 (2012).

42. F. Evers, R. D. L. Hanes, C. Zunke, R. F. Capellmann, J. Bewerunge, C. DalleFerrier, M. C. Jenkins, I. Ladadwa, A. Heuer, R. Castañeda Priego, and S. U. EgelhaAf, Eur. Phys. J. Special Topics (accepted) .

43. D. Wilms, S. Deutschländer, U. Siems, K. Franzrahe, P. Henseler, P. Keim, N. Schwierz, P. Virnau, K. Binder, G. Maret, and P. Nielaba, J. Phys.: Condens. Matter 24, 464119 (2012).

44. C. Kreuter, U. Siems, P. Henseler, P. Nielaba, P. Leiderer, and A. Erbe, $J$. Phys.: Condens. Matter 24, 464120 (2012).

45. A. Malijevský and G. Jackson, J. Phys.: Condens. Matter 24, 464121 (2012).

46. L. Assoud, F. Ebert, P. Keim, R. Messina, G. Maret, and H. Löwen, Phys. Rev. Lett. 102, 238301 (2009).

47. S. Deutschländer, T. Horn, H. Löwen, G. Maret, and P. Keim, Phys. Rev. Lett. 111, 098301 (2013).

48. C. Kreuter, U. Siems, P. Nielaba, P. Leiderer, and A. Erbe, Eur. Phys. J. Special Topics (accepted) .

49. P. Dillmann, G. Maret, and P. Keim, Eur. Phys. J. Special Topics (accepted) .

50. S. Deutschländer, K. Franzrahe, B. Heinze, P. Henseler, P. Keim, N. Schwierz, U. Siems, P. Virnau, D. Wilms, K. Binder, G. Maret, and P. Nielaba, Eur. Phys. J. Special Topics (accepted) .

51. H. Löwen, T. Horn, T. Neuhaus, and B. Ten Hagen, Eur. Phys. J. Special Topics (accepted) .

52. A. Reinmüller, E. C. OĞuz, R. Messina, H. Löwen, H. J. Schöpe, and T. Palberg, Eur. Phys. J. Special Topics (accepted) .

53. E. C. OĞuz, M. Marechal, F. Ramiro-Manzano, I. Rodriguez, R. Messina, F. J. Meseguer, and H. Löwen, Phys. Rev. Lett. 109, 218301 (2012).

54. A. Reinmüller, H. J. Schöpe, and T. Palberg, Soft Matter 6, 5312 (2010).

55. A. Reinmüller, E. C. OĞuz, R. Messina, H. Löwen, H. J. Schöpe, and T. Palberg, J. Chem. Phys 136, 164505 (2012).

56. A. Wysocki, C. P. Royall, R. G. Winkler, G. Gompper, H. Tanaka, A. van BlaAderen, and H. Löwen, Faraday Discuss. 144, 245 (2010).

57. A. Wysocki, C. P. Royall, R. Winkler, G. Gompper, H. Tanaka, A. van BlaAderen, and H. Löwen, Soft Matter 5, 1340 (2009).

58. S. Lang, V. Boţan, M. Oettel, D. Hajnal, T. Franosch, and R. Schilling, Phys. Rev. Lett. 105, 125701 (2010).

59. D. Wilms, A. Winkler, P. Virnau, and K. Binder, Phys. Rev. Lett. 105, 045701 (2010).

60. E. C. OĞuz, R. Messina, and H. Löwen, Europhys. Lett. 94, 28005 (2011).

61. J. Bleibel, S. Dietrich, A. Domínguez, and M. Oettel, Phys. Rev. Lett. 107, 128302 (2011).

62. J. Bleibel, A. Domínguez, and M. Oettel, Eur. Phys. J. Special Topics (accepted).

63. H. H. Wensink, H. Löwen, M. Marechal, A. Härtel, R. Wittkowski, U. Zimmermann, A. Kaiser, and A. M. Menzel, Eur. Phys. J. Special Topics (accepted)

64. T. Schilling, S. Dorosz, M. Radu, M. Mathew, S. Jungblut, and K. Binder, Eur. Phys. J. Special Topics (accepted) .

65. A. B. G. M. Leferink op Reinink, E. van den Pol, A. V. Petukhov, G. J. Vroege, and H. N. W. LekKerkerker, Eur. Phys. J. Special Topics (accepted).

66. E. van den Pol, A. V. Petukhov, D. M. E. Thies-Weesie, D. V. Byelov, and G. J. Vroege, Phys. Rev. Lett. 103, 258301 (2009).

67. S. Meinhardt, J. Smiatek, R. Eichhorn, and F. Schmid, Phys. Rev. Lett. 108, 214504 (2012).

68. R. Wittkowski and H. Löwen, Phys. Rev. E 85, 021406 (2012). 
69. R. Wittkowski and H. Löwen, Molecular Physics 109, 2935 (2011).

70. A. Erbe, M. Zientara, L. Baraban, C. Kreidler, and P. Leiderer, J. Phys.: Condens. Matter 20, 404215 (2008).

71. G. Volpe, I. Buttinoni, D. Vogt, H.-J. Kümmerer, and C. Bechinger, Soft Matter 7, 8810 (2011).

72. F. Kümmel, B. ten Hagen, R. Wittkowski, I. Buttinoni, R. Eichhorn, G. Volpe, H. Löwen, and C. Bechinger, Phys. Rev. Lett. 110, 198302 (2013).

73. I. Buttinoni, J. Bialké, F. Kümmel, H. Löwen, C. Bechinger, and T. Speck, Phys. Rev. Lett. 110, 238301 (2013).

74. H. H. Wensink and H. Löwen, Phys. Rev. E 78, 031409 (2008).

75. Y. Yang, V. Marceau, and G. Gompper, Phys. Rev. E 82, 031904 (2010).

76. A. Kaiser, H. H. Wensink, and H. Löwen, Phys. Rev. Lett. 108, 268307 (2012).

77. H. H. Wensink, J. Dunkel, S. Heidenreich, K. Drescher, R. E. Goldstein, H. Löwen, and J. M. Yeomans, Proc. Natl. Acad. Sci. USA 109, 14308 (2012).

78. J. Bialké, T. Speck, and H. Löwen, Phys. Rev. Lett. 108, 168301 (2012).

79. A. M. Menzel and H. Löwen, Phys. Rev. Lett. 110, 055702 (2013).

80. P. Hänggi and F. Marchesoni, Rev. Mod. Phys. 81, 387 (2009). 